\title{
Oxidative Injury in Ischemic Stroke: A Focus on NADPH Oxidase 4
}

\author{
Ganglei Li, ${ }^{1}$ Changsheng Ye, ${ }^{2}$ Yu Zhu, ${ }^{1}$ Tiesong Zhang, ${ }^{1}$ Jun Gu, ${ }^{1}$ Jianwei Pan, ${ }^{1}$ Feng Wang, \\ Fan Wu, ${ }^{1}$ Kaiyuan Huang, ${ }^{1}$ Kangli $X u,{ }^{1}$ Xiaomin $W u\left(D,{ }^{3}\right.$ and Jian Shen $\mathbb{D}^{1}$ \\ ${ }^{1}$ Department of Neurosurgery, The First Affiliated Hospital, College of Medicine, Zhejiang University, Hangzhou 310003, China \\ ${ }^{2}$ Department of Emergency, Anji County People's Hospital, Huzhou 313300, China \\ ${ }^{3}$ Department of Anesthesiology, Zhejiang Provincial People's Hospital, People's Hospital of Hangzhou Medical College, \\ Hangzhou 310014, China
}

Correspondence should be addressed to Xiaomin Wu; wuxiaomin@hmc.edu.cn and Jian Shen; 1314006@zju.edu.cn

Received 3 July 2021; Revised 8 January 2022; Accepted 17 January 2022; Published 3 February 2022

Academic Editor: Abdur Rauf

Copyright (c) 2022 Ganglei Li et al. This is an open access article distributed under the Creative Commons Attribution License, which permits unrestricted use, distribution, and reproduction in any medium, provided the original work is properly cited.

Ischemic stroke is a leading cause of disability and mortality worldwide. Thus, it is urgent to explore its pathophysiological mechanisms and find new therapeutic strategies for its successful treatment. The relationship between oxidative stress and ischemic stroke is increasingly appreciated and attracting considerable attention. ROS serves as a source of oxidative stress. It is a byproduct of mitochondrial metabolism but primarily a functional product of NADPH oxidases (NOX) family members. Nicotinamide adenine dinucleotide phosphate oxidase 4 (NOX4) is most closely related to the formation of ROS during ischemic stroke. Its expression is significantly upregulated after cerebral ischemia, making it a promising target for treating ischemic stroke. Several drugs targeting NOX4, such as SCM-198, Iso, G-Rb1, betulinic acid, and electroacupuncture, have shown efficacy as treatments of ischemic stroke. MTfp-NOX4 POC provides a novel insight for the treatment of stroke. Combinations of these therapies also provide new approaches for the therapy of ischemic stroke. In this review, we summarize the subcellular location, expression, and pathophysiological mechanisms of NOX4 in the occurrence and development of ischemic stroke. We also discuss the therapeutic strategies and related regulatory mechanisms for treating ischemic stroke. We further comment on the shortcomings of current NOX4-targeted therapy studies and the direction for improvement.

\section{Introduction}

Stroke is a cerebrovascular disease caused by organic brain injury, characterized by abrupt onset and rapid emergence of localized and disseminated deficits in brain function [1-4]. It is a disease with high disability, morbidity, and fatality rates [5-7]. Stroke can be classified as ischemic stroke or hemorrhagic stroke, with ischemic stroke accounting for approximately $80 \%$ of all cases $[8,9]$. Ischemic stroke is a devastating cerebral disease that can cause permanent neurological damage [10]. Although the diagnosis and treatment of ischemic stroke have greatly improved [11-13], the prognosis of patients with ischemic stroke is still poor [14, 15]. Hence, it is necessary to further explore the pathophysiological mechanisms and therapeutic strategies for the treatment of ischemic stroke.
Oxidative stress plays a crucial role in the development and progression of many diseases, especially in ischemic stroke [16-22]. It results from an imbalance between the generation of ROS and antioxidant defense systems. Increased ROS could modify cell structure and enzymatic activity [23-25]. These changes lead to extravasation of blood components, increased inflammatory response, and even irreversible brain tissue damage [26]. Nicotinamide adenine dinucleotide phosphate (NADPH) oxidases (NOXs) and mitochondrial metabolism are the major sources of ROS $[27,28]$. While ROS are byproducts of mitochondrial metabolism, they are functional products of the NOX family. NOXs are composed of the NOX1-5 and DUOX1-2 enzymes and play indispensable roles in cellular homeostasis [29], including gene expression, cell migration, proliferation, aging, and inflammation. NOX4, a member of the NOX family, was first 
identified in the kidney [30]. Growing evidence shows that NOX4 plays a salient role in many diseases. For example, NOX4 was found to function as an oncogene in non-smallcell lung cancer by enhancing glycolysis [31]. In addition, NOX4 facilitates cisplatin-induced acute kidney injury by regulating ROS-mediated programmed cell death and inflammation [32]. Notably, NOX4 is highly expressed in cerebral blood vessels and could contribute to ROS formation after acute cerebral ischemia. Emerging data suggest that NOX4 is significantly associated with ischemic stroke, particularly by increasing levels of oxidative stress [33]. Oxidative stress could contribute to ischemia- and hypoxia-induced brain injury and induce the formation of atherosclerosis, an important cause of ischemic stroke [34, 35]. Importantly, increasing studies demonstrate that the silenced NOX4 impairs BBB leakage, neuronal apoptosis, and oxidative stress. Researches on NOX4-targeted stroke treatment have made significant progress. NOX4 is a promising therapeutic target for treating ischemic stroke.

In this review, we explored basic information about MAGI2-AS3 and its subcellular location and summarized the literature describing the relationship between NOX4 and ischemic stroke, specifically its pathophysiological mechanism. In addition, we discuss therapeutic strategies targeting NOX4 for treating ischemic stroke.

\section{Overview of NOX4: Physiological Function, Subcellular Location, and Expression Patterns}

The NOX4 gene is located on chromosome 11q14, as shown in Figure 1(a). Based on the GeneCard online database (https://www.genecards.org) [36, 37], NOX4 is widely distributed in cells, especially in the plasma membrane, nucleus, and mitochondria, as shown in Figure 1(b). The subcellular localization may be obviously correlated with the function of NOX4. In addition, the expression levels of NOX4 are normally elevated in the normal kidney, artery, lymph node, adipocyte, and breast tissues (Figure 1(c)). NOX4 has a close connection with the function and condition of multiple viscera and systems. More selective targeting of NOX4 is an important direction of stroke-associated researches.

The NOX protein family includes NOX1, NOX2, NOX3, NOX4, NOX5, and the dual oxidases DUOX1 and DUOX2 $[29,38]$. NOX1-5 are expressed in all vascular cell types and play essential roles in many physiological processes, such as endothelial cell function, vascular permeability, and angiogenesis. NOX4 functions as a heme-containing catalytic subunit that contains six transmembrane domains $[39,40]$ and binds p22(phox) to form a heterodimeric enzyme complex [41-43]. Splice variants of the NOX4 gene encode four proteins: NOX4B $(62.7 \mathrm{kDa})$, NOX4C $(25.7 \mathrm{kDa})$, NOX4D $(31.8 \mathrm{kDa})$, and NOX4E $(27.6 \mathrm{kDa})$, of which only the functionally active NOX4D is associated with the production of ROS [44-46]. While NOX4D lacks putative transmembrane domains, it has both NADPH- and flavin adenine dinucleotide-binding domains, which are required for elec- tron transfer activity [45]. Upregulation of NOX4D expression promotes the production of NADPH-dependent ROS and thus enhances the phosphorylation of extracellularsignal-regulated kinase $1 / 2$ and the nuclear transcription factor Elk-1 [45].

Some research groups have reported that NOX1, NOX2, NOX4, and NOX5 exist in all vascular cell types and directly affect many physiological processes, such as endothelial cell function, vascular permeability, and angiogenesis. Under pathological conditions, the expression of NOX4 is dominant in vascular endothelial cells, affecting both their proliferation and angiogenesis by increasing ROS production [47-49].

\section{Pathophysiological Mechanisms of Ischemic Stroke}

Stroke can be classified into ischemic stroke (IS) and hemorrhagic stroke that is characterized by abrupt onset and rapid emergence of localized and disseminated deficits in brain function $[1,2]$. It is a cerebrovascular disease caused by organic brain injury. The most common type of stroke is ischemic stroke $[3,4]$. Ischemic stroke was caused by supplying artery stenosis (the carotid and vertebral arteries) or occlusion or insufficient cerebral blood supply. Four types of ischemic stroke were recognized: TIA (transient ischemic attack), reversible ischemic neurological deficit (RIND), stroke in evolution, and completed stroke [50]. TIA has no infarcts, while RIND, stroke in evolution, and completed stroke have varying cerebral infarcts. Neurons lose their ability to maintain normal transmembrane ionic gradients after ischemia [50] and then cause a series of pathological changes, such as oxidative stress [51], excitotoxicity, inflammation [52], and apoptosis [53]. Increased ROS triggers oxidative stress after ischemia-reperfusion and then aggravates blood-brain barrier injury. These alterations cause blood constituent extravasation, escalate the inflammatory response, and lead to irreversible brain tissue injury [26].

3.1. ROS. Oxidative stress results from an imbalance between ROS generation and antioxidant defense systems [54, 55]. Various types of noxious stimuli lead to excessive production of ROS or reduce the antioxidative capacity and then cause tissue damage. ROS serves as a source of oxidative stress. It is a byproduct of oxygen metabolism but primarily functional products of NOX family members, including free radicals such as $\mathrm{O}_{2}{ }^{-}$and $\mathrm{OH}$ and nonradicals such as $\mathrm{H}_{2} \mathrm{O}_{2}$ $[56,57]$. The production of ROS is stimulated by a series of enzymes, such as cyclooxygenases, myeloperoxidases, and NADPH oxidase [58]. $\mathrm{O}_{2}{ }^{-}$, having less transmembrane capacity, achieves better transmembrane ability via the conversion of $\mathrm{O}_{2}{ }^{-}$to $\mathrm{H}_{2} \mathrm{O}_{2} \cdot \mathrm{H}_{2} \mathrm{O}_{2}$ produces $\mathrm{OH}$ through reacting with $\mathrm{Fe}^{2+}$ to undergo the Fenton reaction $[59,60]$.

Increased ROS could degrade endocellular macromolecules (lipids, proteins, and nucleic acids) and lead to decreased enzymatic activity and alterations of cellular configuration [23-25]. Overproduction of ROS results in a decrease of cell membrane fluidity and permeability by induction of lipid peroxidation. Oxidative stress is not only 


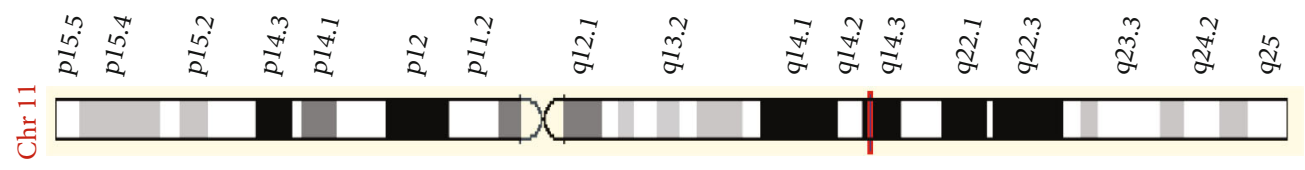

(a)

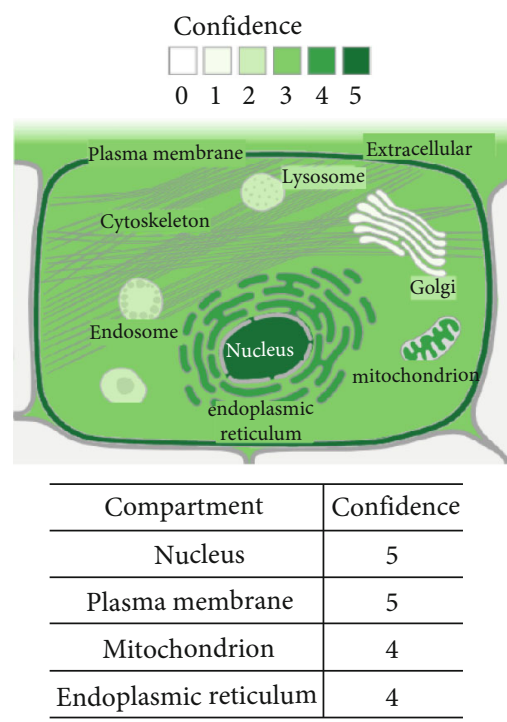

(b)

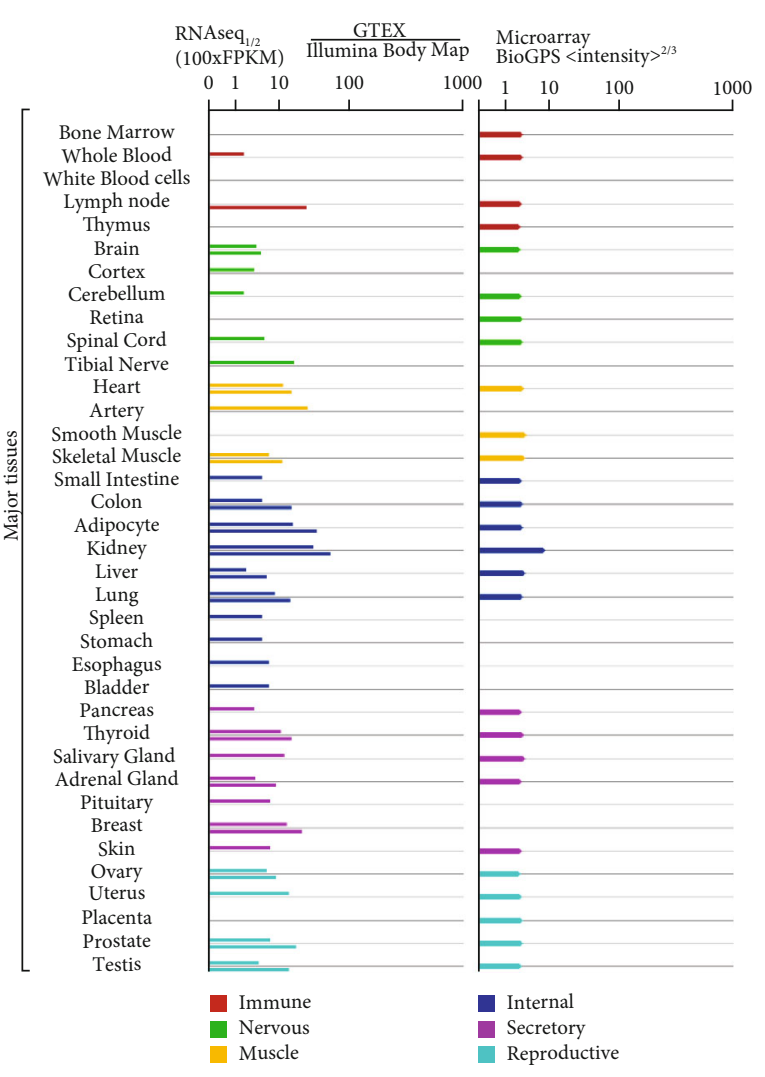

(c)

FIGURE 1: The subcellular localization and gene expression of NOX4 in human tissues. (a) NOX4 gene is located on chromosome 11q14. (b) The expression of NOX4 is concentrated in the nucleus, plasma membrane, mitochondrion, and endoplasmic reticulum. (c) The expression level of NOX4 in normal human tissues.

involved in ischemia- and hypoxia-induced brain injury but was also closely associated with atherosclerosis, an important cause of ischemic stroke $[34,35]$. ROS is known to play an important role in atherosclerosis progression [17, 61]. Risk factors for stroke could lead to vascular endothelial injury and facilitate penetration of LDL into the subendothelial space. LDL is oxidized by ROS [61] and becomes ox-LDL in the subendothelial space. Ox-LDL induces proinflammatory responses and vascular smooth muscle cell proliferation. Activated macrophages produce large levels of ROS by activating NADPH oxidase and then promote the formation of ox-HDL [62]. Finally, atherosclerotic plaque is formed and gradually hardens and narrows the arteries. Atherosclerotic plaque, which is very unstable, could lead to cerebrovascular blockage after the plaque is cracked or ruptured (Figure 2). Large amounts of ROS could induce the early inflammatory response and activate many kinds of immune cells [63]. Then, this triggers the release of numerous cytokines, generates a local effect, and further contributes to more generations of ROS. Oxida- tive stress and inflammation cross-promote each other, thus creating a vicious circle $[64,65]$.

The detection of oxidative stress and inflammatory reaction level potentially could have important clinical implications. Due to the short half-life and highly reactive nature $[66,67]$, it is difficult to detect the ROS level directly. 8Hydroxydeoxyguanosine (8-OHdG) and malondialdehyde (MDA) are products of oxidative DNA damage and lipid oxidative damage and are not affected by metabolic and dietary factors. Hence, $8-\mathrm{OHdG}$ and MDA are potential biomarkers of oxidative stress and could be used for the detection of the oxidative stress level [68-70]. It is an effective approach for the treatment of ischemic stroke by inhibiting ROS production or enhancing the ROS scavenging capacity. The expression levels of antioxidant enzymes were predominantly regulated by Nrf2 [71, 72]. Nrf2 has shown a neuroprotective effect in ischemic stroke [73]. It could be a potential biomarker for the treatment of ischemic stroke $[74,75]$. Butylphthalide has been found to improve the prognosis of ischemic stroke via regulation of Nrf2 [76]. Due to 


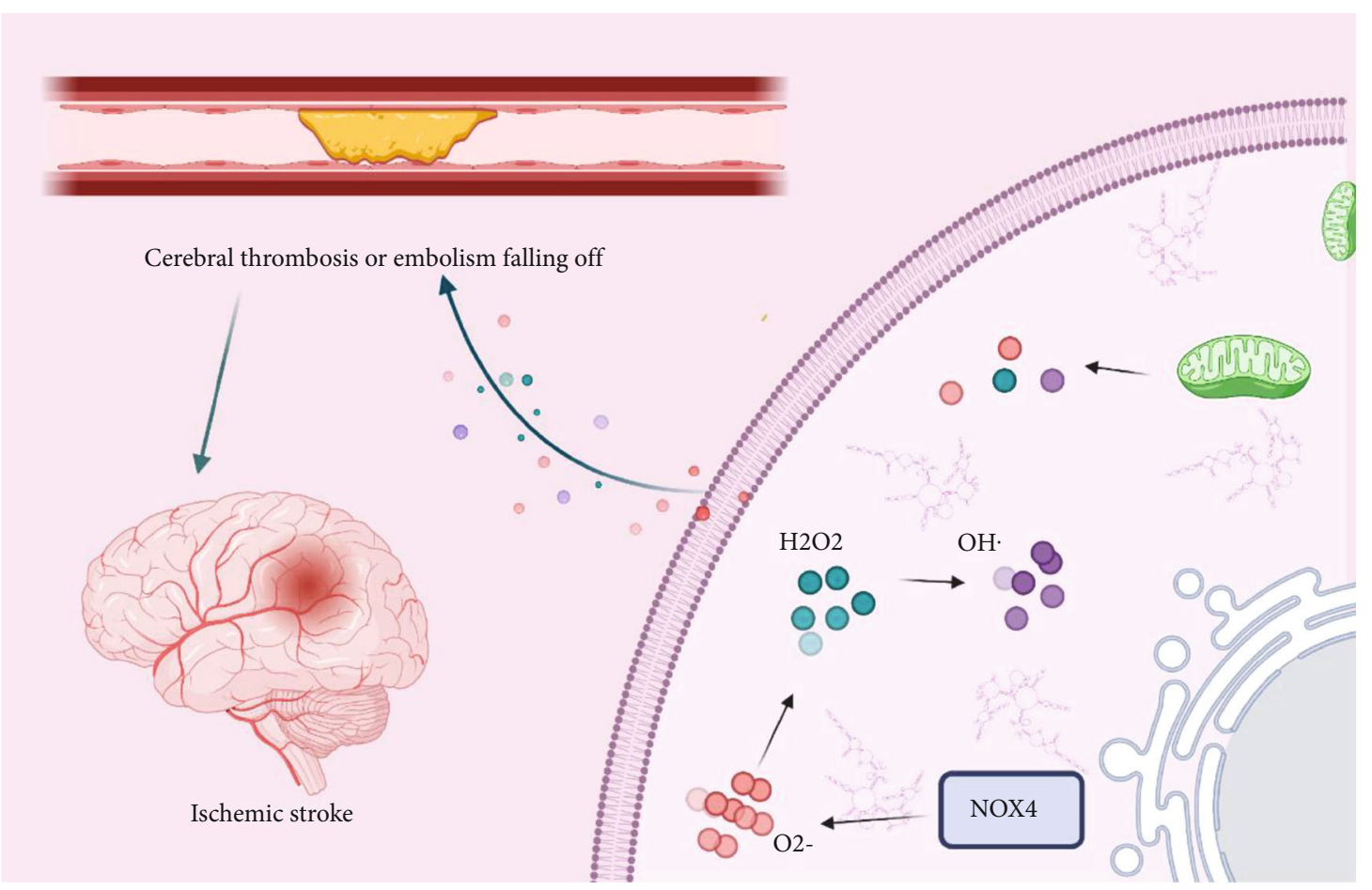

FIGURE 2: ROS serves as a source of oxidative stress. It is a byproduct of oxygen metabolism but primarily functional products of NOX family members, including free radicals such as $\mathrm{O}_{2}^{-}$and $\mathrm{OH}$ and nonradicals such as $\mathrm{H}_{2} \mathrm{O}_{2}$. Oxidative stress was closely associated with atherosclerosis, an important cause of ischemic stroke. Atherosclerotic plaque, very unstable, could lead to cerebrovascular blockage after the plaque is cracked or ruptured.

the interaction between oxidative stress and inflammatory response, detection and interventions of inflammatory reaction could have positive significance for the treatment of ischemic stroke.

3.2. ROS and NOX4. While ROS are byproducts of mitochondrial metabolism, they are primarily functional products of NADPH oxidase (NOX) family members [77, 78]. Growing evidence indicates that NOX is one of the main sources of cellular ROS, including neutrophils and neurons [79-81]. Nox generates ROS by transferring electrons from $\mathrm{NADPH}$ to oxygen, resulting in severe oxidative stress [82, 83]. NOX4 is highly expressed in cerebral blood vessels [84]. Moreover, NOX4 is most closely related to the formation of ROS after acute cerebral ischemia. Dysregulation of NOX4 is involved in a variety of human diseases and was associated with a series of clinicopathological features. NOX4 has great potential as diagnostic biomarkers, prognostic indicators, and therapeutic targets. NOX4-generated ROS has played a significant role in the initiation and development of various human diseases, including ischemic stroke. NOX4 is involved in the onset of atherosclerosis by modulating macrophage cell death induced by oxLDL [85, 86]. On the other hand, oxLDL significantly enhanced NOX4 expression by activating the MEK1/ERK pathway, thereby increasing the production of ROS [87]. NOX4 recruited M2-type tumor-associated macrophages to promote tumor growth via regulating ROS/PI3K axis in nonsmall-cell lung cancer [88]. The role of NOX4 in ischemic stroke is attracting an increasing level of interest. Blockade of NOX4-mediated ROS production and signal transduction pathways may help to improve the prognosis of patients with ischemic stroke.

3.3. NOX4 and the Pathophysiology of Ischemic Stroke. Among NOX family members, NOX4, the most abundant vascular isoform, has shown potential as an important regulator of IR injury. It is significantly induced under hypoxic conditions in different tissues and cells, making it a promising therapeutic target for the treatment of ischemic stroke [89]. Accumulating evidence suggests that NOX4 suppression could reverse the effects of blood-brain barrier (BBB) breakdown, oxidative stress, and neuronal apoptosis during IR injury $[33,90]$. In this section, we will further discuss the role of NOX4 in the pathogenesis of ischemic stroke.

\subsubsection{The Expression and Clinical Features of NOX4 in} Ischemic Stroke. While expression levels of NOX4 are typically elevated in the normal kidney, artery, lymph node, adipocyte, breast, and brain tissue, high NOX4 levels in the brain contribute to ischemic stroke [90]. Some research groups have reported that the expression of NOX4 is high in endothelial cells, astrocytes, and neurons [91]. Further, the major source of NOX4 is endothelial cells in ischemic stroke [92]. In vivo experiments demonstrated that levels of NOX4 are significantly upregulated after cerebral IR injury [90, 93-96]. In addition, the NOX4 rs11018628 polymorphism has been correlated with short-term recovery and decreased risk of ischemic stroke, but the molecular mechanism of this phenomenon remained to be unveiled [97]. 
3.3.2. The Related Mechanisms of NOX4 in Ischemic Stroke. The expression of NOX4 plays a key regulatory role in the development of ischemic stroke. Several studies have revealed that NOX4 leads to ischemic stroke via BBB breakdown and neurodegeneration [33, 90]. Further studies showed that the knockdown of NOX4 impairs BBB leakage, neuronal apoptosis, and oxidative stress [98]. Lou et al. found that activation of the TGF- $\beta$ signaling pathway facilitated the expression of NOX4 by upregulating the expression of ALK5 and SMAD 2/3 in ischemic stroke [99]. The suppression of ALK5 limited the expression of NOX4 and ROS production, resulting in the blockade of cerebral IR injury. The ACE2/ Ang-(1-7)/Mas pathway is also able to ameliorate cerebral injury by downregulating the levels of NOX4 in ischemic stroke [100]. Bioinformatics predictions indicate that miR29c-3p, miR-132-3p, and miR-29a-5p target the NOX4 gene [101]. These microRNAs are thus promising biomarkers for the treatment of ischemic stroke.

\section{Therapy for Ischemic Stroke}

Stroke is a disease with high disability, high morbidity, and high fatality rates with no currently effective therapeutic strategies in the clinic. A growing number of studies have shown that NOX4 levels dramatically correlate with ischemic stroke. With an improved understanding of the pathogenesis and molecular mechanism of ischemic stroke, NOX4 appears to be an important regulator of ischemic stroke pathophysiology. NOX4 is also a promising therapeutic target for the treatment of ischemic stroke. In this section, we will discuss the treatment and related regulatory mechanisms of ischemic stroke (Table 1).

4.1. SCM-198. Leonurine, also called SCM-198, is an alkaloid from the Leonuri cardiacae herba plant that is a promising effective treatment for several diseases [89, 111, 112], such as myocardial infarction, type 2 diabetes, and Alzheimer's disease $[111,113,114]$. Recent evidence indicates that SCM-198 also has potential efficacy against ischemic stroke $[89,102]$. In a rat model of transient middle cerebral artery occlusion (MCAO), SCM-198 could reduce infarct volume and improve neurological deficits by maintaining BBB integrity after ischemic injury [102]. In vitro experiments showed that downregulating ROS production positively correlates with BBB integrity. Additional studies showed that SCM198 facilitates BBB integrity by controlling the HDAC4/ NOX4/MMP-9 axis (Figure 3). Moreover, SCM-198 was shown to play a neuroprotective role by regulating the $\mathrm{p}$ STAT3/NOX4/Bcl-2 pathway, and the p-STAT3 protein was able to bind and regulate NOX4 directly [89]. SCM198 might be an effective new agent for the treatment of cerebral ischemia and reperfusion injury. But the functional roles in vivo need more investigation into ischemic stroke.

4.2. Isoquercetin (Iso). Iso, a novel plant extract [115], exhibits a wide range of therapeutic effects against cancer [116], acute myocardial infarction [117], and diabetes mellitus [118]. Recently, we found that Iso could decrease infarct volume and brain swelling and improve neurological deficits
[73]. Iso inhibits oxidative stress by reducing ROS production and inducing the expression of the $S O D$ and CAT genes. Both in vivo and in vitro experiments showed that Iso elicits antiapoptotic and antioxidant effects via Nrf2-mediated suppression of the NOX4/ROS/NF- $\kappa \mathrm{B}$ axis [73]. Silencing of Nrf2 was found to impair these effects of Iso. Therefore, Iso holds promise as a future treatment of ischemic stroke.

4.3. Ginsenoside Rb1 (G-Rb1). G-Rb1, the major pharmacological extract from ginseng, has been reported to exhibit neuroprotective effects $[103,104]$. In an animal model of MCAO, Chen et al. observed that G-Rb1 could reduce infarct volume, improve neurological deficits, and decrease the degree of cerebral edema. G-Rb1 was also shown to downregulate NOX4 expression and activity in ischemic stroke. Moreover, these authors found that G-Rbl could maintain BBB integrity by inhibiting the neuroinflammationstimulated induction of NOX4- and MMP-9-derived free radicals. However, these studies are limited by the small sample size. Large sample size in vivo research is still needed to further confirm the role of G-Rb1 in ischemic stroke. Additionally, the downstream regulatory mechanism of NOX4 needs to be understood further.

4.4. Electroacupuncture. Electroacupuncture, a type of Chinese medicinal therapy, is increasingly used to treat a variety of diseases, including as an adjuvant treatment for ischemic stroke [119-122]. Jung et al. [105] reported that electroacupuncture decreased infarct volume and improved neurological deficits after ischemic injury. These authors showed that electroacupuncture preconditioning markedly reduced the production of ROS and aquaporin 4 (a BBB component) in astrocytes. The expression of NOX4 was significantly inhibited, while levels of NOX2 were not obviously altered in electroacupuncture-preconditioned mice [105].

4.5. Betulinic Acid. Betulinic acid is a pentacyclic triterpene that is naturally found in birch trees [123-125]. It shows promise as a valuable treatment for cancer [126-128], nonalcoholic fatty liver disease [129], AIDS, and ischemic stroke $[130,131]$. Betulinic acid has been shown to markedly attenuate infarct size and neuronal apoptosis and improve neurological deficits after IR injury [93]. Pretreatment with betulinic acid was shown to downregulate levels of NOX4 and ROS after cerebral ischemia. There are still some limitations in the study of betulinic acid and ischemic stroke. The effectiveness of the treatment was significantly noticed, but not as posttreatment after cerebral ischemia. It was also limited by a short period of observation. The long-term effects of betulinic acid remain unclear.

4.6. Nanomule Peptide Carrier. NOX4 is highly expressed in cerebral blood vessels and could contribute to ROS formation after acute cerebral ischemia. It could be a feasible way to reduce the expression of NOX4 by using NOX4specific siRNA. It has been demonstrated that a twelveamino acid peptide (MTfp), derived from melanotransferrin (MTf), could efficiently transport siRNA through the BBB model by forming a novel peptide-oligonucleotide conjugate (POC) $[106,107]$. MTfp-NOX4 POC further reduced the 
TABLE 1: NOX4-related treatments and its associated mechanisms in ischemic stroke.

\begin{tabular}{|c|c|c|c|}
\hline Drug name & Clinical features & Pathways & Refs \\
\hline SCM-198 & Infarct volume, neurological deficits & HDAC4, NOX4, MMP-9, p-STAT3, and Bcl-2 & {$[89,102]$} \\
\hline Isoquercetin & Infarct volume, brain swelling, and neurological deficits & $\begin{array}{c}\text { ROS, SOD, CAT, Nrf2, NOX4, ROS, and NF- } \\
\kappa \mathrm{B}\end{array}$ & [73] \\
\hline Ginsenoside Rb1 & $\begin{array}{c}\text { Infarct volume, neurological deficits, and the degree of } \\
\text { cerebral edema }\end{array}$ & NOX4 and MMP-9 & {$[103,104]$} \\
\hline Electroacupuncture & Infarct volume and neurological deficits & ROS, aquaporin 4 , and NOX4 & {$[105]$} \\
\hline Betulinic acid & Infarct size, neuronal apoptosis, and neurological deficits & NOX4 and ROS & [93] \\
\hline MTfp-NOX4 POC & Infarct size and neurological deficits & NOX4 & {$[106,107]$} \\
\hline VAS2870 & Neuronal apoptosis and oxidative stress & $\begin{array}{l}\text { NOX2, NOX4, miR-29c-3p, miR-132-3p, and } \\
\text { miR-29a-5p }\end{array}$ & $\begin{array}{c}{[95,98,} \\
101]\end{array}$ \\
\hline Safflor Yellow B & Infarct size, neurological deficits, and motor function & Ak046177, miR-134, NOX4, CREB, and Nrf2 & {$[108]$} \\
\hline Guhong injection & Infarct size and neurological deficits & PKC, HIF- $1 \alpha$, and NOX4 & [109] \\
\hline Melatonin & Neuronal apoptosis and oxidative stress & NOX2 and NOX4 & [91] \\
\hline Apocynin & Neurological deficits & NOX & {$[110]$} \\
\hline
\end{tabular}

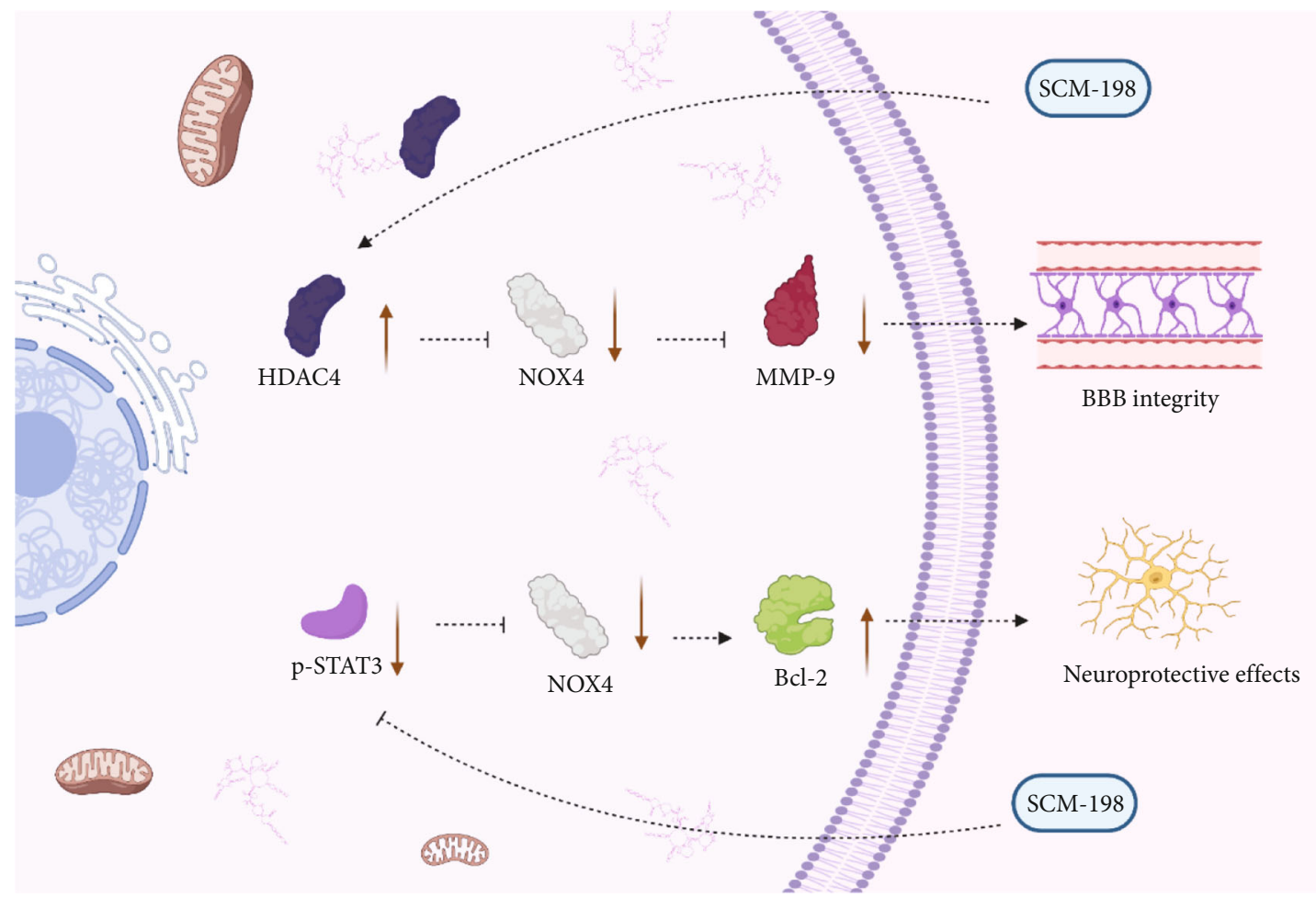

FIGURE 3: The function and molecular mechanism of SCM-198 in ischemic stroke. SCM-198 maintains the integrity of BBB integrity through the regulation of the HDAC4/NOX4/MMP-9 axis. SCM-198 significantly inhibits the expression of NOX4 to downregulate the level of MMP-9 by facilitating the expression of HDAC4. Moreover, SCM-198 plays a neuroprotective role via regulating the p-STAT3/ NOX4/Bcl-2 pathway. SCM-198 promotes the expression of Bcl-2 through downregulating the expression level of p-STAT3 and NOX4.

expression level of NOX4. In vivo experiments showed that silenced NOX4 reduced infarct size and promoted neurological recovery after cerebral infarction. MTfp-NOX4 POC provides a novel insight for the treatment of stroke.

4.7. Other Therapeutic Strategies. Several studies have revealed that VAS2870, a NOX inhibitor, blocks neuronal apoptosis and oxidative stress by targeting NOX2 and NOX4 after ischemic injury $[95,98]$. Intravenous injections of VAS2870 prior to reperfusion appear to significantly upregulate the levels of miR-29c-3p, miR-132-3p, and miR29a-5p, which target NOX4 [101]. Li et al. [91] observed that melatonin may elicit antiapoptotic and antioxidant effects by suppressing the expression of NOX2 and NOX4 after 
cerebral ischemia. Safflor Yellow B, reducing neuronal injury after ischemia-reperfusion, was also involved in NOX4 regulation [108]. Guhong injection could significantly suppress NOX4 expression in the treatment of ischemic stroke [109]. In addition, the NOX inhibitor apocynin was demonstrated to improve neurological deficits in ischemic stroke [110]. Combinations of these therapies may also provide new approaches to treat ischemic stroke [132].

\section{Conclusions and Future Perspectives}

Ischemic stroke is a disease with high disability, high morbidity, and high fatality rates. Despite advancements in cancer treatment techniques, ischemic stroke remains a leading cause of death and disability worldwide. Therefore, it is urgent to find new diagnostic markers and treatments against ischemic stroke. This requires a deeper understanding of ischemic stroke pathophysiology. The crucial role of oxidative stress in the development of ischemic stroke is attracting widespread interest nowadays. Oxidative stress is not only involved in ischemia- and hypoxia-induced brain injury but was also closely associated with atherosclerosis. ROS is the core of oxidative stresses, but it is an unstable compound. So, the detection of stable oxidative stress products, $8-\mathrm{OHdG}$ and MDA, is important for stroke diagnosis and treatment. The regulation of the ROS level may be an effective treatment approach for ischemic stroke. Oxidative stress was significantly associated with inflammatory reaction. Due to the close relationship of oxidative stress and inflammation, detection and interventions of inflammatory reaction may have a certain effect for the treatment of ischemic stroke.

ROS is the byproduct of mitochondrial metabolism but a main functional product of NOX family. In recent years, the role of NOX4 in stroke has been gradually brought to attention. High expression level of NOX4 is most closely related to formation of ROS after acute cerebral ischemia. NOX4 may be a potential diagnostic biomarker. This is an interesting topic, but it is still an emerging field. For clinical application, the biomarker must be stably expressed in body fluids (such as blood or urine or cerebrospinal fluid). Additionally, multicenter clinical research is required. In terms of treatment, clinical research about NOX4 has begun to make progress. The inhibition of NOX4 reversed the effect of blood-brain barrier breakdown, oxidative stress, and neuronal apoptosis during IR injury. Directly or indirectly targeted regulation of NOX4 could control the occurrence and development of ischemic stroke and improve the prognosis of patients. This field remains to be further studied. A promising drug must have the ability to stably regulate the NOX4 level and effectively transduce the effect. To reach this goal, we would need to conduct in-depth research on the structure and function of NOX4. Current research on targeting NOX4 is in its infancy, with many questions remaining. We need to conduct a more thorough analysis of the structure and function of NOX4 under physiological and pathophysiological conditions. In addition, the complex molecular mechanism that regulates the NOX4 network still needs to be further explored.

\section{Conflicts of Interest}

The authors declare that they have no conflicts of interest.

\section{Authors' Contributions}

Ganglei Li and Changsheng Ye contributed equally to this work.

\section{Acknowledgments}

This work was supported by the National Natural Science Foundation of China (82071285) and Basic Public Welfare Research Program of Zhejiang Province (LDG21H090006).

\section{References}

[1] S. Paul and E. Candelario-Jalil, "Emerging neuroprotective strategies for the treatment of ischemic stroke: an overview of clinical and preclinical studies," Experimental Neurology, vol. 335, article 113518, 2021.

[2] S. G. Erkabu, Y. Agedie, D. D. Mihretu, A. Semere, and Y. M. Alemu, "Ischemic and hemorrhagic stroke in Bahir Dar, Ethiopia: a retrospective hospital-based study," Journal of Stroke and Cerebrovascular Diseases, vol. 27, no. 6, pp. 1533-1538, 2018.

[3] X. T. Su, L. Wang, S. M. Ma et al., "Mechanisms of acupuncture in the regulation of oxidative stress in treating ischemic stroke," Oxidative Medicine and Cellular Longevity, vol. 1155, Article ID 7875396, 2020.

[4] S. Sarvari, F. Moakedi, E. Hone, J. W. Simpkins, and X. Ren, "Mechanisms in blood-brain barrier opening and metabolism-challenged cerebrovascular ischemia with emphasis on ischemic stroke," Metabolic Brain Disease, vol. 35, no. 6, pp. 851-868, 2020.

[5] M. Dichgans, S. L. Pulit, and J. Rosand, "Stroke genetics: discovery, biology, and clinical applications," The Lancet Neurology, vol. 18, no. 6, pp. 587-599, 2019.

[6] C. O. Johnson, M. Nguyen, G. A. Roth et al., "Global, regional, and national burden of stroke, 1990-2016: a systematic analysis for the Global Burden of Disease Study 2016," The Lancet Neurology, vol. 18, no. 5, pp. 439-458, 2019.

[7] A. H. Barfejani, M. Jafarvand, S. M. Seyedsaadat, and R. T. Rasekhi, "Donepezil in the treatment of ischemic stroke: review and future perspective," Life Sciences, vol. 263, article 118575, 2020.

[8] S. Xu, J. Lu, A. Shao, J. H. Zhang, and J. Zhang, "Glial cells: role of the immune response in ischemic stroke," Frontiers in Immunology, vol. 11, 2020.

[9] S. Zheng, Y. Y. Bai, Y. Changyi et al., "Multimodal nanoprobes evaluating physiological pore size of brain vasculatures in ischemic stroke models," Advanced Healthcare Materials, vol. 3, no. 11, pp. 1909-1918, 2014.

[10] H. Song, H. Zhou, Z. Qu et al., "From analysis of ischemic mouse brain proteome to identification of human serum clusterin as a potential biomarker for severity of acute ischemic stroke," Translational Stroke Research, vol. 10, no. 5, pp. 546-556, 2019.

[11] F. Erani, N. Zolotova, B. Vanderschelden et al., "Electroencephalography might improve diagnosis of acute stroke and large vessel occlusion," Stroke, vol. 51, no. 11, pp. 3361$3365,2020$. 
[12] A. S. Kim and J. D. Easton, "New opportunities to optimize antithrombotic therapy for secondary stroke prevention," International Journal of Stroke, vol. 14, no. 3, pp. 220-222, 2019.

[13] P. Patel, D. Yavagal, and P. Khandelwal, "Hyperacute Management of Ischemic Strokes:," Journal of the American College of Cardiology, vol. 75, no. 15, pp. 1844-1856, 2020.

[14] G. J. Hankey, "Stroke," The Lancet, vol. 389, no. 10069, pp. 641-654, 2017.

[15] D. Guo, Z. Zhu, C. Zhong et al., "Increased serum netrin-1 is associated with improved prognosis of ischemic stroke," Stroke, vol. 50, no. 4, pp. 845-852, 2019.

[16] J. N. Moloney and T. G. Cotter, "ROS signalling in the biology of cancer," Seminars in Cell \& Developmental Biology, vol. 80, pp. 50-64, 2018.

[17] U. Förstermann, N. Xia, and H. Li, "Roles of vascular oxidative stress and nitric oxide in the pathogenesis of atherosclerosis," Circulation Research, vol. 120, no. 4, pp. 713-735, 2017.

[18] J. Zou, Q. Fei, H. Xiao et al., "VEGF-A promotes angiogenesis after acute myocardial infarction through increasing ROS production and enhancing ER stress-mediated autophagy," Journal of Cellular Physiology, vol. 234, no. 10, pp. 1769017703, 2019.

[19] D. Xian, R. Lai, J. Song, X. Xiong, and J. Zhong, "Emerging perspective: role of increased ROS and redox imbalance in skin carcinogenesis," Oxidative Medicine and Cellular Longevity, vol. 1155, Article ID 8127362, 2019.

[20] J. C. Jha, A. M. D. Watson, G. Mathew, L. C. de Vos, and K. Jandeleit-Dahm, "The emerging role of NADPH oxidase NOX5 in vascular disease," Clinical Science, vol. 131, no. 10, pp. 981-990, 2017.

[21] D. Huang, F. Siaw-Debrah, H. Wang et al., “Transplanting Rac1-silenced bone marrow mesenchymal stem cells promote neurological function recovery in TBI mice," Aging, vol. 13, no. 2, pp. 2822-2850, 2020.

[22] L. Zhang, J. Wu, X. Duan et al., "NADPH oxidase: a potential target for treatment of stroke," Oxidative Medicine and Cellular Longevity, vol. 2016, Article ID 5026984, 2016.

[23] L. A. Tziveleka, M. A. Tammam, O. Tzakou, V. Roussis, and E. Ioannou, "Metabolites with antioxidant activity from marine macroalgae," Antioxidants, vol. 10, no. 9, 2021.

[24] N. Omidifar, A. Nili-Ahmadabadi, A. Nakhostin-Ansari et al., "The modulatory potential of herbal antioxidants against oxidative stress and heavy metal pollution: plants against environmental oxidative stress," Environmental Science and Pollution Research, vol. 28, no. 44, pp. 6190861918, 2021.

[25] F. Wang, X. Zhou, W. Liu et al., "Arsenite-induced ROS/RNS generation causes zinc loss and inhibits the activity of poly(ADP-ribose) polymerase-1," Free Radical Biology \& Medicine, vol. 61, pp. 249-256, 2013.

[26] R. He, Y. Jiang, Y. Shi, J. Liang, and L. Zhao, "Curcuminladen exosomes target ischemic brain tissue and alleviate cerebral ischemia-reperfusion injury by inhibiting ROSmediated mitochondrial apoptosis," Materials Science and Engineering: C, vol. 117, article 111314, 2020.

[27] C. Canugovi, M. D. Stevenson, A. E. Vendrov et al., "Increased mitochondrial NADPH oxidase 4 (NOX4) expression in aging is a causative factor in aortic stiffening," Redox Biology, vol. 26, article 101288, 2019.
[28] S. Drevet, G. Gavazzi, L. Grange, C. Dupuy, and B. Lardy, "Reactive oxygen species and NADPH oxidase 4 involvement in osteoarthritis," Experimental Gerontology, vol. 111, pp. 107-117, 2018.

[29] A. Tarafdar and G. Pula, "The role of NADPH oxidases and oxidative stress in neurodegenerative disorders," International Journal of Molecular Sciences, vol. 19, no. 12, 2018.

[30] K. Bedard and K. H. Krause, "The NOX family of ROSgenerating NADPH oxidases: physiology and pathophysiology," Physiological Reviews, vol. 87, no. 1, pp. 245-313, 2007.

[31] C. Zeng, Q. Wu, J. Wang et al., "NOX4 supports glycolysis and promotes glutamine metabolism in non-small cell lung cancer cells," Free Radical Biology \& Medicine, vol. 101, pp. 236-248, 2016.

[32] X. M. Meng, G. L. Ren, L. Gao et al., "NADPH oxidase 4 promotes cisplatin-induced acute kidney injury via ROSmediated programmed cell death and inflammation," Laboratory Investigation, vol. 98, no. 1, pp. 63-78, 2018.

[33] K. A. Radermacher, K. Wingler, F. Langhauser et al., "Neuroprotection after stroke by targeting NOX4 as a source of oxidative stress," Antioxidants \& Redox Signaling, vol. 18, no. 12, pp. 1418-1427, 2013.

[34] N. Khatri, M. Thakur, V. Pareek, S. Kumar, S. Sharma, and A. K. Datusalia, "Oxidative stress: major threat in traumatic brain injury," CNS \& Neurological Disorders Drug Targets, vol. 17, no. 9, pp. 689-695, 2018.

[35] W. Xu, T. Li, L. Gao et al., "Apelin-13/APJ system attenuates early brain injury via suppression of endoplasmic reticulum stress-associated TXNIP/NLRP3 inflammasome activation and oxidative stress in a AMPK-dependent manner after subarachnoid hemorrhage in rats," Journal of Neuroinflammation, vol. 16, no. 1, p. 247, 2019.

[36] J. W. Yu, L. Wang, and L. D. Bao, "Exploring the active compounds of traditional Mongolian medicine in intervention of novel coronavirus (COVID-19) based on molecular docking method," Journal of Functional Foods, vol. 71, article 104016, 2020.

[37] Y. Liu, Y. Ma, Z. Li et al., "Investigation of inhibition effect of gossypol-acetic acid on gastric cancer cells based on a network pharmacology approach and experimental Validation," Drug Design, Development and Therapy, vol. 14, pp. 3615$3623,2020$.

[38] F. Augsburger, A. Filippova, D. Rasti et al., "Pharmacological characterization of the seven human NOX isoforms and their inhibitors," Redox Biology, vol. 26, article 101272, 2019.

[39] S. Dikalov, A. Dikalova, A. Bikineyeva, H. Schmidt, D. Harrison, and K. Griendling, "Distinct roles of Nox1 and Nox4 in basal and angiotensin II-stimulated superoxide and hydrogen peroxide production," Free Radical Biology \& Medicine, vol. 45, no. 9, pp. 1340-1351, 2008.

[40] S. P. Gray, A. M. Shah, and I. Smyrnias, "NADPH oxidase 4 and its role in the cardiovascular system," Vascular Biology, vol. 1, no. 1, pp. H59-h66, 2019.

[41] K. von Löhneysen, D. Noack, M. R. Wood, J. S. Friedman, and U. G. Knaus, "Structural insights into Nox4 and Nox2: motifs involved in function and cellular localization," Molecular and Cellular Biology, vol. 30, no. 4, pp. 961975,2010

[42] K. von Löhneysen, D. Noack, A. J. Jesaitis, M. C. Dinauer, and U. G. Knaus, "Mutational analysis reveals distinct features of 
the Nox4-p22 phox complex," The Journal of Biological Chemistry, vol. 283, no. 50, pp. 35273-35282, 2008.

[43] T. Kawahara and J. D. Lambeth, "Molecular evolution of Phox-related regulatory subunits for NADPH oxidase enzymes," BMC Evolutionary Biology, vol. 7, no. 1, 2007.

[44] J. N. Moloney, A. K. Jayavelu, J. Stanicka et al., "Nuclear membrane-localised NOX4D generates pro-survival ROS in FLT3-ITD-expressing AML," Oncotarget, vol. 8, no. 62, pp. 105440-105457, 2017.

[45] N. Anilkumar, G. S. Jose, I. Sawyer et al., “A 28-kDa splice variant of NADPH oxidase-4 is nuclear-localized and involved in redox signaling in vascular cells," Arteriosclerosis, Thrombosis, and Vascular Biology, vol. 33, no. 4, pp. e104e112, 2013.

[46] P. Goyal, N. Weissmann, F. Rose et al., "Identification of novel Nox4 splice variants with impact on ROS levels in A549 cells," Biochemical and Biophysical Research Communications, vol. 329, no. 1, pp. 32-39, 2005.

[47] A. B. García-Redondo, A. Aguado, A. M. Briones, and M. Salaices, "NADPH oxidases and vascular remodeling in cardiovascular diseases," Pharmacological Research, vol. 114, pp. 110-120, 2016.

[48] F. Rezende, F. Moll, M. Walter et al., "The NADPH organizers NoxO1 and p47phox are both mediators of diabetesinduced vascular dysfunction in mice," Redox Biology, vol. 15, pp. 12-21, 2018.

[49] S. P. Gray and K. A. Jandeleit-Dahm, "The role of NADPH oxidase in vascular disease-hypertension, atherosclerosis \& stroke," Current Pharmaceutical Design, vol. 21, no. 41, pp. 5933-5944, 2015.

[50] S. Kaithoju, "Ischemic stroke: risk stratification, warfarin teatment and outcome measure," Journal of Atrial Fibrillation, vol. 8, no. 4, p. 1144, 2015.

[51] P. Chazelas, C. Steichen, F. Favreau et al., "Oxidative stress evaluation in ischemia reperfusion models: characteristics, limits and perspectives," International Journal of Molecular Sciences, vol. 22, no. 5, p. 2366, 2021.

[52] W. Li, Y. Zhang, Z. Hu, and Y. Xu, "Overexpression of NLRC3 enhanced inhibition effect of sevoflurane on inflammation in an ischaemia reperfusion cell model," Folia Neuropathologica, vol. 58, no. 3, pp. 213-222, 2020.

[53] Z. Lv, C. Liu, M. Zhai et al., "LPS pretreatment attenuates cerebral ischaemia/reperfusion injury by inhibiting inflammation and apoptosis," Cellular Physiology and Biochemistry, vol. 45, no. 6, pp. 2246-2256, 2018.

[54] A. van der Pol, W. H. van Gilst, A. A. Voors, and P. van der Meer, "Treating oxidative stress in heart failure: past, present and future," European Journal of Heart Failure, vol. 21, no. 4, pp. 425-435, 2019.

[55] P. A. Mouthuy, S. J. Snelling, S. G. Dakin et al., "Biocompatibility of implantable materials: an oxidative stress viewpoint," Biomaterials, vol. 109, pp. 55-68, 2016.

[56] M. Chvanov, O. H. Petersen, and A. Tepikin, "Free radicals and the pancreatic acinar cells: role in physiology and pathology," Philosophical Transactions of the Royal Society B: Biological Sciences, vol. 360, no. 1464, pp. 2273-2284, 2005.

[57] M. Valko, D. Leibfritz, J. Moncol, M. T. Cronin, M. Mazur, and J. Telser, "Free radicals and antioxidants in normal physiological functions and human disease," The International
Journal of Biochemistry \& Cell Biology, vol. 39, no. 1, pp. 44-84, 2007.

[58] H. N. Siti, Y. Kamisah, and J. Kamsiah, "The role of oxidative stress, antioxidants and vascular inflammation in cardiovascular disease (a review)," Vascular Pharmacology, vol. 71, pp. 40-56, 2015.

[59] Y. Gao, J. Duan, X. Zhai et al., "Photocatalytic degradation and antibacterial properties of Fe3+-Doped alkalized carbon nitride," Nanomaterials, vol. 10, no. 9, 2020.

[60] O. Sabido, A. Figarol, J. P. Klein et al., "Quantitative flow cytometric evaluation of oxidative stress and mitochondrial impairment in RAW 264.7 macrophages after exposure to pristine, acid functionalized, or annealed carbon nanotubes," Nanomaterials, vol. 10, no. 2, 2020.

[61] A. J. Kattoor, N. V. K. Pothineni, D. Palagiri, and J. L. Mehta, "Oxidative stress in atherosclerosis," Current Atherosclerosis Reports, vol. 19, no. 11, 2017.

[62] S. Y. Kim, J. M. Jeong, S. J. Kim et al., "Pro-inflammatory hepatic macrophages generate ROS through NADPH oxidase 2 via endocytosis of monomeric TLR4-MD2 complex," Nature Communications, vol. 8, no. 1, p. 2247, 2017.

[63] J. Park, J. S. Min, B. Kim et al., "Mitochondrial ROS govern the LPS-induced pro-inflammatory response in microglia cells by regulating MAPK and NF- $\kappa \mathrm{B}$ pathways," Neuroscience Letters, vol. 584, pp. 191-196, 2015.

[64] T. Hussain, B. Tan, Y. Yin, F. Blachier, M. C. Tossou, and N. Rahu, "Oxidative stress and inflammation: what polyphenols can do for us?," Oxidative Medicine and Cellular Longevity, vol. 1155, Article ID 7432797, 2016.

[65] V. Singh and S. Ubaid, "Role of silent information regulator 1 (SIRT1) in regulating oxidative stress and inflammation," Inflammation, vol. 43, no. 5, pp. 1589-1598, 2020.

[66] H. A. Sawal, K. Asghar, M. Bureik, and N. Jalal, "Bystander signaling via oxidative metabolism," Oncotargets and Therapy, vol. 10, pp. 3925-3940, 2017.

[67] H. Deng, Z. Zhou, W. Yang et al., "Endoplasmic reticulum targeting to amplify immunogenic cell death for cancer immunotherapy," Nano Letters, vol. 20, no. 3, pp. 19281933, 2020.

[68] M. Graille, P. Wild, J. J. Sauvain, M. Hemmendinger, I. Guseva Canu, and N. B. Hopf, "Urinary 8-OHdG as a biomarker for oxidative stress: a systematic literature review and meta-analysis," International Journal of Molecular Sciences, vol. 21, no. 11, p. 3743, 2020.

[69] L. J. Kroese and P. G. Scheffer, "8-Hydroxy-2'-deoxyguanosine and cardiovascular disease: a systematic review," Current Atherosclerosis Reports, vol. 16, no. 11, p. 452, 2014.

[70] D. Tsikas, "Assessment of lipid peroxidation by measuring malondialdehyde (MDA) and relatives in biological samples: analytical and biological challenges," Analytical Biochemistry, vol. 524, pp. 13-30, 2017.

[71] Z. L. Song, F. Bai, B. Zhang, and J. Fang, "Synthesis of dithiolethiones and identification of potential neuroprotective agents via activation of Nrf2-driven antioxidant enzymes," Journal of Agricultural and Food Chemistry, vol. 68, no. 7, pp. 2214-2231, 2020.

[72] S. Peng, Y. Hou, J. Yao, and J. Fang, "Activation of Nrf2driven antioxidant enzymes by cardamonin confers neuroprotection of PC12 cells against oxidative damage," Food \& Function, vol. 8, no. 3, pp. 997-1007, 2017. 
[73] Y. Dai, H. Zhang, J. Zhang, and M. Yan, "Isoquercetin attenuates oxidative stress and neuronal apoptosis after ischemia/ reperfusion injury via Nrf2-mediated inhibition of the NOX4/ROS/NF- $\kappa \mathrm{B}$ pathway," Chemico-Biological Interactions, vol. 284, pp. 32-40, 2018.

[74] T. Yang, Y. Sun, Q. Li et al., "Ischemic preconditioning provides long-lasting neuroprotection against ischemic stroke: the role of Nrf2," Experimental Neurology, vol. 325, article $113142,2020$.

[75] M. Liu, H. Li, L. Zhang et al., "Cottonseed oil alleviates ischemic stroke-induced oxidative stress injury via activating the Nrf2 signaling pathway," Molecular Neurobiology, vol. 58, no. 6, pp. 2494-2507, 2021.

[76] Y. Huang, L. Pan, and T. Wu, "Improvement of cerebral ischemia-reperfusion injury by L-3-n-butylphthalide through promoting angiogenesis," Experimental Brain Research, vol. 239, no. 1, pp. 341-350, 2021.

[77] V. Helfinger, K. Palfi, A. Weigert, and K. Schröder, "The NADPH oxidase Nox4 controls macrophage polarization in an NFאB-dependent manner," Oxidative Medicine and Cellular Longevity, vol. 2019, Article ID 3264858, 2019.

[78] S. Khalid, H. Yamazaki, M. Socorro, D. Monier, E. Beniash, and D. Napierala, "Reactive oxygen species (ROS) generation as an underlying mechanism of inorganic phosphate $\left(\mathrm{P}_{\mathrm{i}}\right)$ induced mineralization of osteogenic cells," Free Radical Biology \& Medicine, vol. 153, pp. 103-111, 2020.

[79] R. P. Brandes, N. Weissmann, and K. Schröder, "Nox family NADPH oxidases: molecular mechanisms of activation," Free Radical Biology \& Medicine, vol. 76, pp. 208-226, 2014.

[80] M. A. Khan, L. M. Philip, G. Cheung et al., "Regulating NETosis: increasing $\mathrm{pH}$ promotes NADPH oxidasedependent NETosis," Frontiers in Medicine, vol. 5, 2018.

[81] M. Olguín-Albuerne and J. Morán, "Redox signaling mechanisms in nervous system development," Antioxidants \& Redox Signaling, vol. 28, no. 18, pp. 1603-1625, 2018.

[82] Y. Maejima, J. Kuroda, S. Matsushima, T. Ago, and J. Sadoshima, "Regulation of myocardial growth and death by NADPH oxidase," Journal of Molecular and Cellular Cardiology, vol. 50, no. 3, pp. 408-416, 2011.

[83] M. V. Nguyen, B. Lardy, F. Rousset et al., "Quinone compounds regulate the level of ROS production by the NADPH oxidase Nox4," Biochemical Pharmacology, vol. 85, no. 11, pp. 1644-1654, 2013.

[84] S. Basuroy, S. Bhattacharya, C. W. Leffler, and H. Parfenova, "Nox4 NADPH oxidase mediates oxidative stress and apoptosis caused by TNF-alpha in cerebral vascular endothelial cells," American Journal of Physiology. Cell Physiology, vol. 296, no. 3, pp. C422-C432, 2009.

[85] P. Hu, X. Wu, A. R. Khandelwal et al., "Endothelial Nox4-based NADPH oxidase regulates atherosclerosis via soluble epoxide hydrolase," Biochimica et Biophysica Acta (BBA) - Molecular Basis of Disease, vol. 1863, no. 6, pp. 1382-1391, 2017.

[86] S. Guo and X. Chen, "The human Nox4: gene, structure, physiological function and pathological significance," Journal of Drug Targeting, vol. 23, no. 10, pp. 888-896, 2015.

[87] C. F. Lee, M. Qiao, K. Schröder, Q. Zhao, and R. Asmis, "Nox4 is a novel inducible source of reactive oxygen species in monocytes and macrophages and mediates oxidized low density lipoprotein-induced macrophage death," Circulation Research, vol. 106, no. 9, pp. 1489-1497, 2010.
[88] J. Zhang, H. Li, Q. Wu et al., "Tumoral NOX4 recruits M2 tumor-associated macrophages via ROS/PI3K signalingdependent various cytokine production to promote NSCLC growth," Redox Biology, vol. 22, article 101116, 2019.

[89] Q. Y. Zhang, Z. J. Wang, L. Miao et al., "Neuroprotective effect of SCM-198 through stabilizing endothelial cell function," Oxidative Medicine and Cellular Longevity, vol. 2019, Article ID 7850154, 2019.

[90] A. I. Casas, E. Geuss, P. W. Kleikers et al., "NOX4-dependent neuronal autotoxicity and BBB breakdown explain the superior sensitivity of the brain to ischemic damage," Proceedings of the National Academy of Sciences of the United States of America, vol. 114, no. 46, pp. 12315-12320, 2017.

[91] H. Li, Y. Wang, D. Feng et al., "Alterations in the time course of expression of the Nox family in the brain in a rat experimental cerebral ischemia and reperfusion model: effects of melatonin," Journal of Pineal Research, vol. 57, no. 1, pp. 110-119, 2014.

[92] P. W. Kleikers, V. T. Dao, E. Göb et al., "SFRR-E Young Investigator Awardee NOXing out stroke: Identification of NOX4 and 5 as targets in blood-brain-barrier stabilisation and neuroprotection," Free Radical Biology \& Medicine, vol. 75, 2014.

[93] P. Lu, C. C. Zhang, X. M. Zhang et al., "Down-regulation of NOX4 by betulinic acid protects against cerebral ischemiareperfusion in mice," Current Medical Science, vol. 37, no. 5, pp. 744-749, 2017.

[94] S. K. McCann, G. J. Dusting, and C. L. Roulston, "Early increase of Nox4 NADPH oxidase and superoxide generation following endothelin-1-induced stroke in conscious rats," Journal of Neuroscience Research, vol. 86, no. 11, pp. 25242534, 2008.

[95] Y. H. Tuo, Z. Liu, J. W. Chen et al., "NADPH oxidase inhibitor improves outcome of mechanical reperfusion by suppressing hemorrhagic transformation," Journal of $\mathrm{Neu}$ rointerventional Surgery, vol. 9, no. 5, pp. 492-498, 2017.

[96] C. J. Taylor, R. M. Weston, G. J. Dusting, and C. L. Roulston, "NADPH oxidase and angiogenesis following endothelin-1 induced stroke in rats: role for nox 2 in brain repair," Brain Sciences, vol. 3, no. 4, pp. 294-317, 2013.

[97] W. He, Q. Wang, L. Gu, L. Zhong, and D. Liu, "NOX4 rs11018628 polymorphism associates with a decreased risk and better short-term recovery of ischemic stroke," Experimental and Therapeutic Medicine, vol. 16, no. 6, pp. 52585264, 2018.

[98] C. Kleinschnitz, H. Grund, K. Wingler et al., "Post-stroke inhibition of induced NADPH oxidase type 4 prevents oxidative stress and neurodegeneration," PLoS Biology, vol. 8, no. 9, 2010.

[99] Z. Lou, A. P. Wang, X. M. Duan et al., "Upregulation of NOX2 and NOX4 mediated by TGF- $\beta$ signaling pathway exacerbates cerebral ischemia/reperfusion oxidative stress injury," Cellular Physiology and Biochemistry : International Journal of Experimental Cellular Physiology, Biochemistry, and Pharmacology, vol. 46, no. 5, pp. 2103-2113, 2018.

[100] J. L. Zheng, G. Z. Li, S. Z. Chen et al., “Angiotensin converting enzyme 2/Ang-(1-7)/mas axis protects brain from ischemic injury with a tendency of age-dependence," CNS Neuroscience \& Therapeutics, vol. 20, no. 5, pp. 452-459, 2014.

[101] Z. Liu, Y. H. Tuo, J. W. Chen et al., "NADPH oxidase inhibitor regulates microRNAs with improved outcome after mechanical reperfusion," Journal of Neurointerventional Surgery, vol. 9, no. 7, pp. 702-706, 2017. 
[102] Q. Y. Zhang, Z. J. Wang, D. M. Sun et al., "Novel therapeutic effects of leonurine on ischemic stroke: new mechanisms of BBB integrity," Oxidative Medicine and Cellular Longevity, vol. 2017, Article ID 7150376, 2017.

[103] X. Kang, W. Hong, K. Xie et al., "Ginsenoside Rb1 pretreatment reverses hippocampal changes in BDNF/TrkB mRNA and protein in rats subjected to acute immobilization stress," Drug Design, Development and Therapy, vol. 13, pp. 21272134, 2019.

[104] W. Chen, Y. Guo, W. Yang, P. Zheng, J. Zeng, and W. Tong, "Protective effect of ginsenoside Rb1 on integrity of bloodbrain barrier following cerebral ischemia," Experimental Brain Research, vol. 233, no. 10, pp. 2823-2831, 2015.

[105] Y. S. Jung, S. W. Lee, J. H. Park, H. B. Seo, B. T. Choi, and H. K. Shin, "Electroacupuncture preconditioning reduces ROS generation with NOX4 down-regulation and ameliorates blood-brain barrier disruption after ischemic stroke," Journal of Biomedical Science, vol. 23, no. 1, 2016.

[106] B. A. Eyford, C. S. Singh, T. Abraham et al., "A nanomule peptide carrier delivers siRNA across the intact blood-brain barrier to attenuate ischemic stroke," Frontiers in Molecular Biosciences, vol. 8, article 611367, 2021.

[107] B. A. Eyford, C. S. B. Singh, T. Abraham et al., "Corrigendum: a nanomule peptide carrier delivers sirna across the intact blood-brain barrier to attenuate ischemic stroke," Frontiers in Molecular Biosciences, vol. 8, article 687587, 2021.

[108] C. Wang, H. Wan, Q. Wang et al., "Safflor yellow B attenuates ischemic brain injury via downregulation of long noncoding AK046177 and inhibition of microRNA-134 expression in rats," Oxidative Medicine and Cellular Longevity, vol. 2020, Article ID 4586839, 2020.

[109] L. Yu, Y. Zhang, X. Zhao, H. Wan, Y. He, and W. Jin, "Guhong injection alleviates cerebral ischemia-reperfusion injury via the PKC/HIF- $1 \alpha$ pathway in rats," Frontiers in Pharmacology, vol. 12, article 716121, 2021.

[110] Y. Y. Qin, M. Li, X. Feng et al., "Combined NADPH and the NOX inhibitor apocynin provides greater anti-inflammatory and neuroprotective effects in a mouse model of stroke," Free Radical Biology \& Medicine, vol. 104, pp. 333-345, 2017.

[111] Z. Y. Hong, S. S. Yu, Z. J. Wang, and Y. Z. Zhu, "SCM-198 ameliorates cognitive deficits, promotes neuronal survival and enhances $\mathrm{CREB} / \mathrm{BDNF} / \mathrm{TrkB}$ signaling without affecting $\mathrm{A} \beta$ burden in $\mathrm{A} \beta \mathrm{PP} / \mathrm{PS} 1$ mice," International Journal of Molecular Sciences, vol. 16, no. 8, article 18544, 63 pages, 2015.

[112] Q. Zhu, W. Cai, X. Sha et al., "Quantification of leonurine, a novel potential cardiovascular agent, in rat plasma by liquid chromatography-tandem mass spectrometry and its application to pharmacokinetic study in rats," Biomedical Chromatography: BMC, vol. 26, no. 4, pp. 518-523, 2012.

[113] H. Huang, H. Xin, X. Liu et al., "Novel anti-diabetic effect of SCM-198 via inhibiting the hepatic NF- $\kappa \mathrm{B}$ pathway in $d b / d b$ mice," Bioscience Reports, vol. 32, no. 2, pp. 185195, 2012.

[114] S. Luo, S. Xu, J. Liu, F. Ma, and Y. Z. Zhu, "Design and synthesis of novel SCM-198 analogs as cardioprotective agents: structure-activity relationship studies and biological evaluations," European Journal of Medicinal Chemistry, vol. 200, article 112469, 2020.

[115] T. Lu, C. Zhang, M. Chai, and Y. An, "Isoquercetin ameliorates tunicamycin-induced apoptosis in rat dorsal root ganglion neurons via suppressing ROS-dependent endoplasmic reticulum stress," Biomedicine \& Pharmacotherapy, vol. 80, pp. 343-351, 2016.

[116] J. I. Zwicker, B. L. Schlechter, J. D. Stopa et al., “Targeting protein disulfide isomerase with the flavonoid isoquercetin to improve hypercoagulability in advanced cancer," JCI Insight, vol. 4, no. 4, 2019.

[117] H. Cao, H. Xu, G. Zhu, and S. Liu, "Isoquercetin ameliorated hypoxia/reoxygenation-induced H9C2 cardiomyocyte apoptosis via a mitochondrial-dependent pathway," Biomedicine \& Pharmacotherapy, vol. 95, pp. 938-943, 2017.

[118] M. Jayachandran, T. Zhang, K. Ganesan, B. Xu, and S. S. M. Chung, "Isoquercetin ameliorates hyperglycemia and regulates key enzymes of glucose metabolism via insulin signaling pathway in streptozotocin-induced diabetic rats," European Journal of Pharmacology, vol. 829, pp. 112-120, 2018.

[119] Z. Liu, Y. Liu, H. Xu et al., "Effect of electroacupuncture on urinary leakage among women with stress urinary incontinence: a randomized clinical trial," JAMA, vol. 317 , no. 24 , pp. 2493-2501, 2017.

[120] J. Y. Park and U. Namgung, "Electroacupuncture therapy in inflammation regulation: current perspectives," Journal of Inflammation Research, vol. 11, pp. 227-237, 2018.

[121] S. S. Ng, W. W. Leung, T. W. Mak et al., "Electroacupuncture reduces duration of postoperative ileus after laparoscopic surgery for colorectal cancer," Gastroenterology, vol. 144, no. 2, pp. 307-313.e1, 2013.

[122] Y. G. Huang, W. Tao, S. B. Yang, J. F. Wang, Z. G. Mei, and Z. T. Feng, "Autophagy: novel insights into therapeutic target of electroacupuncture against cerebral ischemia/reperfusion injury," Neural Regeneration Research, vol. 14, no. 6, pp. 954-961, 2019.

[123] J. L. Ríos and S. Máñez, "New pharmacological opportunities for betulinic acid," Planta Medica, vol. 84, no. 1, pp. 8-19, 2018.

[124] X. Wang, X. Lu, R. Zhu et al., "Betulinic acid induces apoptosis in differentiated PC12 cells via ROS-mediated mitochondrial pathway," Neurochemical Research, vol. 42, no. 4, pp. 1130-1140, 2017.

[125] A. Saneja, D. Arora, R. Kumar, R. D. Dubey, A. K. Panda, and P. N. Gupta, "Therapeutic applications of betulinic acid nanoformulations," Annals of the New York Academy of Sciences, vol. 1421, no. 1, pp. 5-18, 2018.

[126] D. Lee, S. R. Lee, K. S. Kang et al., "Betulinic acid suppresses ovarian cancer cell proliferation through induction of apoptosis," Biomolecules, vol. 9, no. 7, p. 257, 2019.

[127] D. M. Zhang, H. G. Xu, L. Wang et al., "Betulinic acid and its derivatives as potential antitumor agents," Medicinal Research Reviews, vol. 35, no. 6, pp. 1127-1155, 2015.

[128] D. Dutta, B. Paul, B. Mukherjee et al., "Nanoencapsulated betulinic acid analogue distinctively improves colorectal carcinoma in vitro and in vivo," Scientific Reports, vol. 9, no. 1, 2019.

[129] Q. Mu, H. Wang, L. Tong et al., "Betulinic acid improves nonalcoholic fatty liver disease through YY1/FAS signaling pathway," The FASEB Journal, vol. 34, no. 9, pp. 1303313048, 2020.

[130] C. Aiken and C. Chen, "Betulinic acid derivatives as HIV-1 antivirals," Trends in Molecular Medicine, vol. 11, no. 1, pp. 31-36, 2005. 
[131] Q. X. Huang, H. F. Chen, X. R. Luo, Y. X. Zhang, X. Yao, and $\mathrm{X}$. Zheng, "Structure and anti-HIV activity of betulinic acid analogues," Current Medical Science, vol. 38, no. 3, pp. 387397, 2018.

[132] A. I. Casas, A. A. Hassan, S. J. Larsen et al., "From single drug targets to synergistic network pharmacology in ischemic stroke," Proceedings of the National Academy of Sciences of the United States of America, vol. 116, no. 14, pp. 71297136, 2019. 